\title{
ORIGINAL
}

\section{CARACTERÍSTICAS DEMOGRÁFICAS, HÁBITOS DE VIDA E HISTORIA DEL CONSUMO DE TABACO DE LOS FUMADORES OCASIONALES EN ESPAÑA (*)}

Lucía Díez-Gañán, Fernando Rodríguez-Artalejo, José Ramón Banegas Banegas, Pilar Guallar-Castillón, Luis Fernández Pacheco y Juan del Rey Calero

Departamento de Medicina Preventiva y Salud Pública. Facultad de Medicina. Universidad Autónoma de Madrid. (*) Durante la realización de este trabajo Lucía Díez Gañán ha disfrutado de una beca BEFI del Fondo de Investigación Sanitaria (Instituto de Salud Carlos III).

\section{RESUMEN}

Fundamento: El consumo ocasional de tabaco suele ser un estado de transición hacia otros patrones de consumo más intenso o frecuente. Este trabajo describe las características sociodemográficas, los hábitos de vida relacionados con la salud, y la historia de consumo de tabaco de los fumadores ocasionales en España, y examina si éstos presentan diferencias con los que consumen tabaco a diario.

Métodos: Los datos se tomaron de la Encuesta Nacional de Salud de España realizada en 1993 mediante entrevista en los domicilios a una muestra representativa de la población española no institucionalizada de 16 y más años. Los análisis se realizaron mediante regresión logística múltiple ajustando por variables sociodemográficas, de estado de salud y hábitos de vida.

Resultados: De los 6.668 fumadores de la encuesta, los fumadores ocasionales representaron el $9,2 \%$, mientras que los fumadores diarios de $\leq 5$ cigarrillos y de $>5$ cigarrillos fueron respectivamente el $9,9 \%$ y el $80,9 \%$. En comparación con los fumadores diarios de $>5$ cigarrillos, los fumadores ocasionales fueron con más frecuencia mujeres (odds ratio [0R]): 2,12 IC95\%:1,72-2,61), más jóvenes (OR de edad 25-44 frente a 16-24 años: 0,75 ; 0,58-0,96), tuvieron menor consumo de alcohol ( $\mathrm{p}$ de tendencia lineal: 0,0349 ) e hicieron más actividad en tiempo libre ( $\mathrm{p}$ tendencia lineal: $<0,0001$ ). Los fumadores ocasionales consumían menos cigarrillos el día que lo hacían que los fumadores diarios $(\mathrm{p}<0,0001)$. Los fumadores ocasionales eran más frecuentes entre los fumadores jóvenes (menos de 20 años) con duraciones relativamente breves de consumo de tabaco (menos de 3 años), y entre los de más edad (65 y más años) que habían fumado durante más tiempo (más de 50 años). Los fumadores diarios de $\leq 5$ cigarrillos presentaron características intermedias entre los ocasionales y los diarios de $>5$ cigarrillos.

Conclusiones. Los fumadores ocasionales tienen características sociodemográficas, hábitos de vida e historia de consumo de tabaco diferentes a los que consumen tabaco diariamente. Estas diferencias sugieren que deben realizarse programas de investigación e intervención dirigidos de forma específica a los fumadores ocasionales.

Palabras clave: Tabaco. Fumadores ocasionales. España

Correspondencia:

Dr. Fernando Rodríguez-Artalejo

Departamento de Medicina Preventiva y Salud Pública

Facultad de Medicina

Universidad Autónoma de Madrid

Avda. Arzobispo Morcillo, s/n

28029 MADRID

Correo electrónico: fernando.artalejo@uam.es

\section{ABSTRACT}

Demographic characteristics, health-related lifestyle, and history of tobacco consumption of the occasional smokers in Spain

Background. This study describes the sociodemographic characteristics, health-related lifestyle, and history of tobacco consumption of the occasional smokers in Spain, and examines whether they show differences against daily smokers.

Methods. Data were obtained from the National Health Survey of Spain, carried out in 1993 through household interviews on a sample representative of the non-institutionalised population aged 16 year and older. Analyses were performed with logistic regression and adjusted for sociodemographic, health-state and life-style variables.

Results. Out of the 6,668 smokers in the survey, occasional smokers were $9.2 \%$, while daily smokers of $\leq 5$ cigarettes and $>5$ cigarettes were $9.9 \%$ and $80.9 \%$, respectively. As compared with daily smokers of $>5$ cigarettes, occasional smokers were more frequently women (odds ratio (0R):2.12; CI95\%:1.72-2.61), younger (OR aged $25-44$ versus $16-24$ years: $0.75 ; 0.58-0.96$ ), with lower alcohol consumption ( $\mathrm{p}$ for linear trend: 0.0349 ), and higher leisure-time physical activity ( $\mathrm{p}$ for linear trend: $<0.0001$ ). On the day they smoke, occasional smokers used to consume less cigarettes than daily smokers $(\mathrm{p}<0.0001)$. Occasional smokers were more frequent among young smokers (aged less than 20) with relatively short history of tobacco consumption (less than 20 years), and also among older smokers (aged 65 year and older) with longer history of consumption (over 50 years). Daily smokers of $\leq 5$ cigarettes showed characteristics midway between those of occasional and daily smokers of $>5$ cigarettes.

Conclusions: Occasional smokers show sociodemographic characteristics, health-related lifestyle, and history of tobacco consumption different from daily smokers. Such differences suggest that research and intervention programs specifically tailored to occasional smokers should be developed.

Keywords: Tobacco. Occasional smokers. Spain 


\section{INTRODUCCIÓN}

La nicotina es una sustancia con gran poder adictivo ${ }^{1}$. No obstante, algunos fumadores parecen presentar una menor dependencia al tabaco, teniendo un consumo diario relativamente bajo (e.g., 5 cigarrillos o menos) ${ }^{2}$ o un consumo no diario u ocasional ${ }^{3}$. El consumo ocasional de tabaco suele ser un estado de transición hacia otros patrones de consumo más intenso o frecuente. Se trata de los fumadores que se inician en el consumo, los que intentan dejarlo, y los que recaen después de haberlo abandonado ${ }^{4}$. Sin embargo, parece haber una pequeña proporción de fumadores que tienen un consumo ocasional durante periodos de tiempo muy prolongados $^{3}$. La tasa de éxito de cesación tabáquica de los fumadores ocasionales es mayor que la de los fumadores diarios, tanto en los jóvenes ${ }^{5}$ como en los de mayor edad ${ }^{6}$, probablemente por la menor dependencia nicotínica de los fumadores ocasionales ${ }^{3}$. A pesar de ello, existen evidencias de que también el consumo ocasional, y no sólo el consumo diario, se asocia a riesgos para la salud, en concreto, mayor mortalidad total y cardiovascular $^{7}$. Además, la duración del consumo es un factor de riesgo de morbimortalidad más importante que su intensidad ${ }^{8}$.

La prevalencia de fumadores ocasionales en la población general mayor de 16 años oscila entre un $3,7 \%$ en California ${ }^{4}$, un $6 \%$ en Finlandia ${ }^{7}$ y un $7 \%$ en Canadá 9 , y alcanza el $18 \%$ entre los jóvenes universitarios de EEUU $^{10}$. Se ha estimado que $5-10 \%$ de los fumadores consumen 5 o menos cigarrillos al día, al menos 4 días a la semana². En España se han registrado prevalencias tan altas como las de Canadá, concretamente del 7,2\% en la población de 14-70 años de Castilla y León en $1994^{11}$. Y a nivel nacional, la prevalencia de fumadores ocasionales en España fue del $4 \%$ en $1993^{12}$. La prevalencia fue mayor en las mujeres que en los hombres hasta los 45 años de edad, y disminuyó con la edad en los dos sexos. Entre los 16 y 17 años, el 27,6\% de los fumadores y el $40,7 \%$ de las fumadoras lo eran de forma ocasional. Más reciente- mente en Gran Canaria ${ }^{13}$ se ha observado que un $38 \%$ de los consumidores de tabaco de 16 a 24 años eran fumadores ocasionales. Sin embargo, en España, a diferencia de otros países como Estados Unidos de Améri $\mathrm{ca}^{3,4,14,15}$ o Suecia ${ }^{16}$, no se han examinado todavía las características sociodemográficas de los fumadores ocasionales ni su asociación con el grado de consolidación del hábito tabáquico.

Los hábitos de vida relacionados con la salud están estrechamente relacionados entre sí y con frecuencia varios hábitos nocivos se presentan de forma simultánea en el mismo sujeto, identificándose conglomerados de dichos estilos de vida ${ }^{17-19}$. Dada la naturaleza multicausal de enfermedades crónicas, como las cardiovasculares o el cáncer, el efecto de la combinación de estilos de vida puede ser sinérgico más que aditivo para el riesgo de enfermar ${ }^{20-22}$. La asociación del consumo de tabaco con otros hábitos de vida no saludables (mayor consumo de alcohol, menor actividad física), está bien documentada ${ }^{17-19,23-26}$, pero en España no se ha examinado todavía si hay diferencias en los hábitos de vida relevantes para la salud entre los distintos tipos de fumadores.

Por todo lo anterior, este trabajo describe las características sociodemográficas, los hábitos de vida relacionados con la salud, y la historia de consumo de tabaco de los fumadores ocasionales en España, y examina si presentan diferencias con las de otros tipos de fumadores. Esta información puede orientar el diseño de intervenciones más específicas para el control del tabaquismo ocasional y de otros patrones de consumo de tabaco que reduzcan el riesgo de enfermedades crónicas.

\section{SUJETOS Y MÉTODO}

\section{Sujetos}

Los datos proceden de las Encuesta $\mathrm{Na}$ cional de Salud de España (ENSE) de 
1993 realizada sobre una muestra aleatoria representativa de la población española no institucionalizada de 16 y más años de edad, estratificada por sexo, edad y tamaño del municipio de residencia ${ }^{12}$. La información se obtuvo mediante entrevista en el domicilio, que completaron 19.384 sujetos, con una tasa de respuesta global para todas las variables de interés del $91,9 \%$.

\section{Variables}

Se recogió información sobre las siguientes variables: 1) sociodemográficas: edad, sexo, nivel de estudios, situación laboral, estado civil, apoyo social y tamaño del municipio de residencia, 2) estado de salud del sujeto: presencia de enfermedades crónicas y salud subjetiva, 3) características e historia del consumo de tabaco: frecuencia del consumo, número de cigarrillos consumidos, edad a la que empezó a fumar y 4) otros hábitos de vida: consumo de alcohol, actividad física en el tiempo libre y actividad física en el trabajo.

Para determinar el consumo de tabaco se realizó la siguiente pregunta: «¿Podría usted decirme si fuma?», con las siguientes respuestas posibles: 1) Sí, fumo a diario; 2) Sí fumo, pero no a diario; 3) No fumo, pero he fumado; 4) No fumo, ni he fumado nunca de manera habitual. Los sujetos que responden la alternativa 1 son considerados fumadores diarios, los que responden la 2 fumadores ocasionales, los de la 3 exfumadores, y los que refieren la 4 nunca fumadores. Para conocer la intensidad del consumo se preguntó a los fumadores diarios: «¿Qué cantidad de cigarrillos fuma usted por término medio al día?», y a los fumadores ocasionales: «Con qué frecuencia suele usted fumar?», con tres posibles respuestas: 1) Tres o cuatro veces por semana, 2) Una o dos veces por semana, 3) Con menos frecuencia (menos de una vez por semana); y «¿qué cantidad de cigarrillos fuma el día que fuma?». Para determinar la edad de inicio se realizó la siguiente pregunta a todos los fumadores actuales (diarios y ocasionales): «¿a qué edad comenzó usted a fumar?». La duración del hábito se obtuvo mediante la diferencia entre la edad actual del sujeto y la edad a la que comenzó a fumar. Según la frecuencia e intensidad de su hábito tabáquico se distinguieron tres tipos de sujetos: los que fuman diariamente $\leq 5$ cigarrillos, los que fuman diariamente $>5$ cigarrillos, y los fumadores ocasionales.

\section{Análisis de datos}

Las diferencias sociodemográficas, en el estado de salud, en las características del hábito tabáquico y en otros hábitos de vida entre los sujetos que fuman ocasionalmente, los que fuman $\leq 5$ cigarrillos/día y los que fuman $>5$ cigarrillos/día se contrastaron con la prueba de chi cuadrado cuando eran variables cualitativas, y con la t de Student cuando eran cuantitativas y su distribución era normal. En caso contrario, las variables cuantitativas se compararon con la prueba de Wilcoxon.

La asociación independiente entre el patrón de consumo de tabaco y las variables de interés se resumió mediante odds ratios ajustados obtenidos mediante modelos de regresión logística múltiple no condicionada. Se construyeron dos modelos. En el primero la variable dependiente fue el consumo ocasional de tabaco frente al consumo diario de $>5$ cigarrillos/día. En el segundo, la variable dependiente fue el consumo ocasional frente al consumo diario de $\leq 5$ cigarrillos/día. En los dos modelos se utilizaron las mismas variables independientes: sociodemográficas (sexo, edad, nivel de estudios, estado civil, apoyo social, situación laboral y tamaño del municipio de residencia), el estado de salud del sujeto (salud subjetiva y presencia de enfermedades crónicas), y hábitos de vida distintos al consumo de tabaco (consumo de alcohol, activi- 
dad física en el trabajo y actividad física en el tiempo libre).

Las variables independientes se introdujeron en los modelos como términos indicadores (dummies): sexo (varón, mujer), edad (16-24, 25-44, 45-64 y $\geq 65$ años), nivel de estudios ( $\sin$ estudios, estudios primarios, estudios secundarios, estudios universitarios), estado civil (casado, no casado), apoyo social (vive acompañado, vive solo), situación laboral (trabaja, jubilado o pensionista, en paro, estudiante, otras situaciones), tamaño del municipio ( $\leq 100.000,100.001$ $400.000,>400.000$ habitantes), salud subjetiva (óptima, subóptima) ${ }^{27}$, presencia de enfermedades crónicas (sí/no), consumo de alcohol (no bebedor, 1-30 g/semana, 31-60 $\mathrm{g} / \mathrm{semana}, \geq 61 \mathrm{~g} / \mathrm{semana}^{28}$, actividad física en el trabajo (sedentario, baja, moderada, elevada/intensa) ${ }^{29}$, actividad física en tiempo libre (sedentario, baja, moderada, elevada/intensa ${ }^{29}$. La enfermedad crónica incluye hipertensión arterial, hipercolesterolemia, diabetes, asma o bronquitis crónica, enfermedad del corazón, úlcera de estómago y alergias. Por último, para estudiar las relaciones dosis-respuesta las variables de interés se han introducido en los modelos en forma continua.

Los análisis se han realizado con el paquete estadístico $\mathrm{SAS}^{30}$.

\section{RESULTADOS}

De los 19.384 sujetos de 16 y más años entrevistados en 1993, $10.021(51,7 \%)$ no fumaban o no habían fumado nunca de manera habitual; $2695(13,9 \%)$ eran exfumadores y $6.668(34,4 \%)$ eran fumadores. La prevalencia de consumo ocasional de tabaco fue $3,2 \%$, y la de consumo diario $31,2 \%(3,4 \%$ fuma $\leq 5$ cigarrillos/día, y $27,8 \%$ fuma $>5$ cigarrillos/día).

La tabla 1 presenta las características demográficas y del estado de salud, algunos hábitos de vida, y las características del consumo de tabaco de los fumadores, según su patrón de consumo. Los fumadores ocasionales representaban el 9,2\% de todos los fumadores, mientras que los fumadores diarios de $\leq 5$ cigarrillos y de $>5$ cigarrillos eran respectivamente el $9,9 \%$ y el $80,9 \%$. La mayoría de los fumadores ocasionales tenían menos de 44 años, estudios primarios o secundarios, vivían acompañados, estaban empleados, residían en ciudades de más de 400.000 habitantes, presentaban salud subjetiva óptima y carecían de enfermedades crónicas. Además eran abstemios o consumían $30 \mathrm{~g}$ o menos de alcohol al día, y su actividad física predominante durante el trabajo era baja, siendo sedentarios durante el tiempo libre. La mayoría de ellos empezó a fumar hacía 5 años o menos, iniciándose en el hábito cuando tenían 16 años o menos. Prácticamente todos ellos fumaban menos de 5 cigarrillos el día que lo hacían.

En comparación con los fumadores diarios de $>5$ cigarrillos, los fumadores ocasionales eran en mayor proporción mujeres, eran más jóvenes, con mayor nivel de estudios, con más frecuencia no estaban casados y eran estudiantes. Además, consumían menos alcohol y eran más sedentarios en el trabajo y menos en el tiempo libre. Por último, se habían iniciado más tarde en el consumo de tabaco, y la duración y magnitud del consumo era menor (tabla 1). En comparación con los fumadores diarios de $\leq 5$ cigarrillos, los fumadores ocasionales eran más jóvenes, con más frecuencia tenían estudios secundarios, no estaban casados, eran estudiantes y tenían salud subjetiva óptima. También eran algo más sedentarios en el trabajo pero menos en el tiempo libre, y empezaron a fumar un poco más tarde, siendo la duración y magnitud de su consumo de tabaco menor (tabla 1). En general, los fumadores diarios de $\leq 5$ cigarrillos presentaron características intermedias entre los ocasionales y los diarios de $>5$ cigarrillos. 
Tabla 1

Características sociodemográficas, de estado de salud, hábitos de vida relacionados con la salud e historia del consumo de tabaco de los fumadores según su patrón de consumo de tabaco. España, 1993

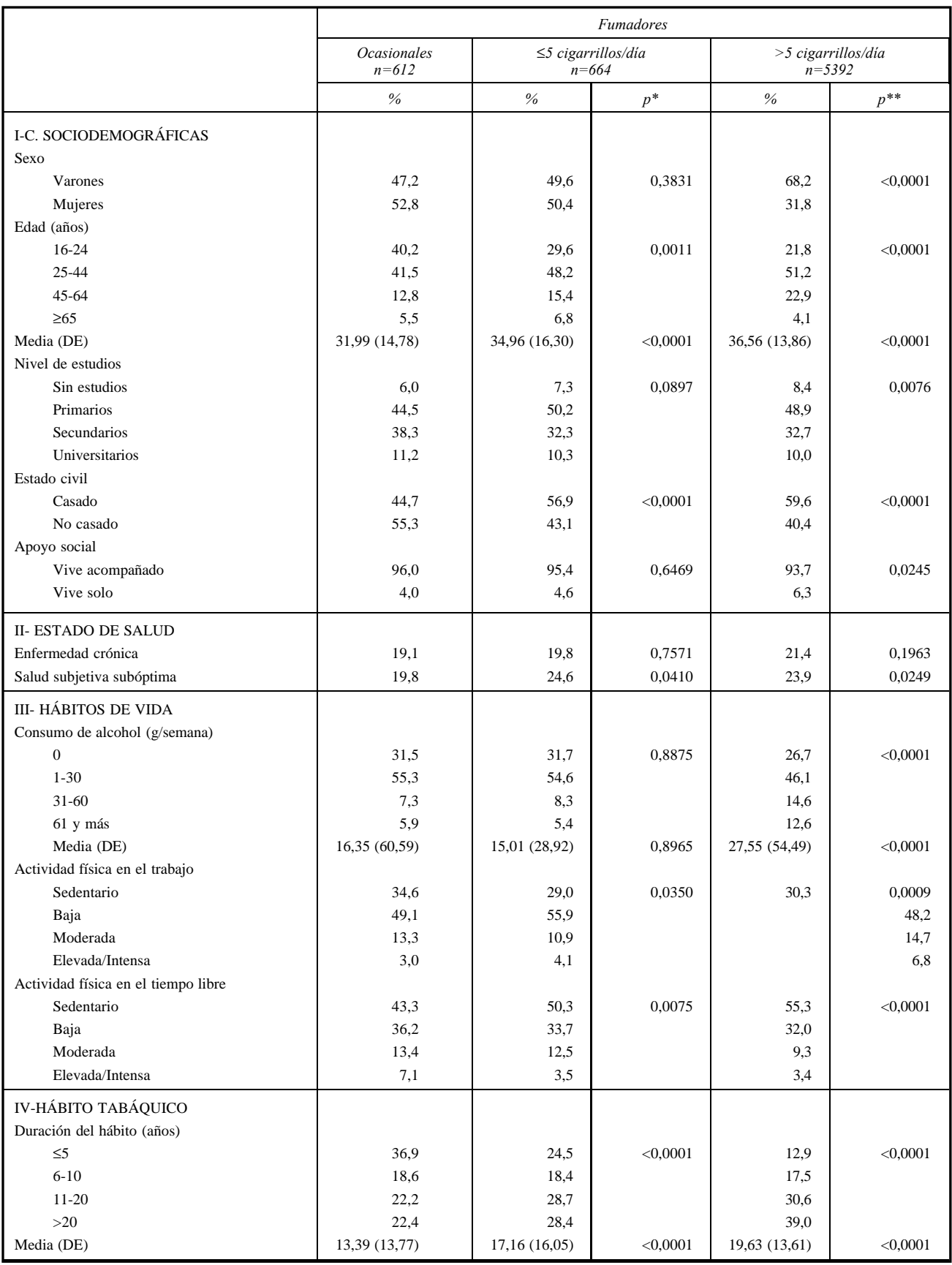


Tabla 1

Características sociodemográficas, de estado de salud, hábitos de vida relacionados con la salud e historia del consumo de tabaco de los fumadores según su patrón de consumo de tabaco. España, 1993

\begin{tabular}{|l|c|r|r|r|r|}
\hline \multirow{2}{*}{} & \multicolumn{4}{|c|}{ Fumadores } \\
\cline { 2 - 6 } & $\begin{array}{c}\text { Ocasionales } \\
n=612\end{array}$ & \multicolumn{2}{|c|}{$\begin{array}{c}\text { s cigarrillos/dia } \\
n=664\end{array}$} & \multicolumn{2}{|c|}{$>5$ cigarrillos/dia } \\
$n=5392$
\end{tabular}

$\mathrm{n}=6668$ fumadores; *p: nivel de significación al comparar a los fumadores de $\leq 5$ cigarrillos/día frente a los fumadores ocasionales; **p: nivel de significación al comparar a los fumadores de > 5 cigarrillos/día frente a los fumadores ocasionales; $\dagger$ En el caso de los fumadores ocasionales: cigarrillos/vez, es decir, número de cigarrillos que fuma el día que lo hace.

La tabla 2 presenta los resultados de los modelos de regresión logística múltiple. Después de ajustar simultáneamente por todas las variables de interés, los fumadores ocasionales fueron con más frecuencia mujeres, más jóvenes, no casados, con menor consumo de alcohol y más actividad en tiempo libre que los fumadores diarios de $>5$ cigarrillos. Además, en comparación con los fumadores diarios de $\leq 5$ cigarrillos, los ocasionales fueron con más frecuencia no casados y realizaban actividad física elevada/intensa en el tiempo libre.

La figura 1 muestra la distribución de los fumadores según el patrón de consumo y la duración del hábito o la edad del fumador. La frecuencia de fumadores ocasionales era mayor entre los sujetos con consumos de tabaco de duración relativamente breve (menos de 3 años) y entre los que habían fumado muchos años (más de 50 años). Sin embargo, incluso en duraciones intermedias del hábito tabáquico había una proporción sustancial de fumadores ocasionales que como promedio fue el $7 \%$ de todos los fumadores. Los fumadores diarios de $\leq 5$ cigarrillos presentaban una distribución similar, mientras que los fumadores diarios de $>5$ cigarrillos presentaban la inversa (figura 1a). La distribución de los fumadores fue prácticamente igual cuando en lugar de la duración del consumo de tabaco se utilizó la edad del fumador (figura 1b).

\section{DISCUSIÓN}

Los fumadores españoles, clasificados según el patrón de consumo de tabaco, son un grupo heterogéneo en sus características sociodemográficas, hábitos de vida relevantes para la salud e historia de consumo de tabaco. En particular, este trabajo muestra que los fumadores ocasionales son una proporción importante de todos los fumadores, son más jóvenes, con más frecuencia mujeres, y con hábitos de vida más saludables que los fumadores diarios. Además, los fumadores ocasionales se inician más tarde en el consumo de tabaco, y la duración e intensidad del mismo es menor, que la de los diarios. Por último, los fumadores diarios de $\leq 5$ cigarrillos presentan en general características intermedias entre los fumadores ocasionales y los diarios de $>5$ cigarrillos.

Rev Esp Salud Pública 2002, Vol. 76, N. ${ }^{\circ} 4$ 
Tabla 2

Odds ratios (OR) ajustadas de ser fumador ocasional frente a ser fumador $\leq \mathbf{5}$ cigarrillos/día (Modelo 1$)$ y de ser fumador ocasional frente a ser fumador $>5$ cigarrillos/día (Modelo 2), para características sociodemográficas, estado de salud, y hábitos de vida relacionados con la salud. España, 1993

\begin{tabular}{|c|c|c|}
\hline & $\begin{array}{c}\text { Modelo 1: Fumador ocasional frente } \\
\text { a fumador } \leq 5 \text { cig./dia }\end{array}$ & $\begin{array}{c}\text { Modelo 2: Fumador ocasional frente } \\
\text { a fumador }>5 \text { cig./dia }\end{array}$ \\
\hline & OR (IC 95\%) & OR (IC 95\%) \\
\hline \multicolumn{3}{|c|}{ I-CARÁCTERÍSTICAS SOCIODEMOGRÁFICAS } \\
\hline \multicolumn{3}{|l|}{ Sexo } \\
\hline Varones & 1,00 & 1,00 \\
\hline Mujeres & $1,07(0,81-1,42)$ & $2,12(1,72-2,61) \ddagger$ \\
\hline \multicolumn{3}{|l|}{ Edad } \\
\hline $16-24$ & 1,00 & 1,00 \\
\hline $25-44$ & $0,99(0,70-1,40)$ & $0,75(0,58-0,96) \dagger$ \\
\hline $45-64$ & $1,18(0,72-1,92)$ & $0,67(0,47-0,95) \dagger$ \\
\hline$\geq 65$ & $1,38(0,58-3,27)$ & $1,27(0,68-2,36)$ \\
\hline \multicolumn{3}{|l|}{ Nivel de estudios } \\
\hline Sin estudios & 1,00 & 1,00 \\
\hline Primarios & $0,95(0,58-1,55)$ & $1,03(0,71-1,53)$ \\
\hline Secundarios & $1,10(0,65-1,86)$ & $1,02(0,68-1,55)$ \\
\hline Universitarios & $1,06(0,58-1,96)$ & $1,01(0,63-1,63)$ \\
\hline \multicolumn{3}{|l|}{ Estado civil } \\
\hline Casado & 1,00 & 1,00 \\
\hline No casado & $1,48(1,07-2,05) \dagger$ & $1,29(1,01-1,64) \dagger$ \\
\hline \multicolumn{3}{|l|}{ Apoyo social } \\
\hline Vive acompañado & 1,00 & 1,00 \\
\hline Vive solo & $0,77(0,43-1,40)$ & $0,65(0,40-1,01)$ \\
\hline \multicolumn{3}{|l|}{ II-ESTADO DE SALUD } \\
\hline \multicolumn{3}{|c|}{ Presencia de enfermedad crónica } \\
\hline No & 1,00 & 1,00 \\
\hline Sí & $1,17(0,85-1,61)$ & $1,03(0,82-1,30)$ \\
\hline \multicolumn{3}{|l|}{ Salud subjetiva } \\
\hline Óptima & 1,00 & 1,00 \\
\hline Subóptima & $0,80(0,60-1,08)$ & $0,81(0,64-1,01)$ \\
\hline \multicolumn{3}{|l|}{ III-HÁBITOS DE VIDA } \\
\hline \multicolumn{3}{|c|}{ Consumo de alcohol (g/semana) } \\
\hline 0 & 1,00 & 1,00 \\
\hline $1-30$ & $0,95(0,74-1,24)$ & $0,97(0,80-1,19)$ \\
\hline $31-60$ & $0,92(0,57-1,47)$ & $0,57(0,40-0,81) \ddagger$ \\
\hline 61 y más & $1,16(0,68-1,98)$ & $0,60(0,40-0,87) \ddagger$ \\
\hline $\mathrm{p}$ de tendencia lineal & 0,4724 & 0,0349 \\
\hline \multicolumn{3}{|c|}{ Actividad física en el trabajo } \\
\hline Sedentario & 1,00 & 1,00 \\
\hline Baja & $0,90(0,68-1,20)$ & $1,15(0,92-1,44)$ \\
\hline Moderada & $1,31(0,87-1,97)$ & $1,18(0,87-1,59)$ \\
\hline Elevada/Intensa & $0,83(0,42-1,62)$ & $0,80(0,46-1,31)$ \\
\hline \multicolumn{3}{|c|}{ Actividad física en el tiempo libre } \\
\hline Sedentario & 1,00 & 1,00 \\
\hline Baja & $1,17(0,91-1,52)$ & $1,30(1,07-1,58) \ddagger$ \\
\hline Moderada & $0,95(0,65-1,39)$ & $1,60(1,19-2,14) \ddagger$ \\
\hline Elevada/Intensa & $2,07(1,17-3,64) \dagger$ & $2,56(1,74-3,70) \ddagger$ \\
\hline $\mathrm{p}$ de tendencia lineal & 0,0930 & $<0,0001$ \\
\hline
\end{tabular}

Modelo 1: OR de ser fumador ocasional frente a ser fumador de $\leq 5$ cigarrillos/día, ajustado por sexo, edad actual, nivel de estudios, estado civil, apoyo social, situación laboral, tamaño del hábitat, estado de salud (presencia de enfermedad crónica y salud subjetiva) y hábitos de vida relacionados con la salud (consumo de alcohol, actividad física en el trabajo y actividad física en el tiempo libre).

Modelo 2: OR de ser fumador ocasional frente a ser fumador de >5 cigarrillos/día, ajustado por sexo, edad actual, nivel de estudios, estado civil, apoyo social, situación laboral, tamaño del hábitat, estado de salud (presencia de enfermedad crónica y salud subjetiva) y hábitos de vida relacionados con la salud (consumo de alcohol, actividad física en el trabajo y actividad física en el tiempo libre). $† \mathrm{p}<0,05 ; \neq \mathrm{p}<0,01$. 


\section{Figura 1}

Distribución de los fumadores según el patrón de consumo y la duración del hábito de fumar o la edad del fumador

1a)

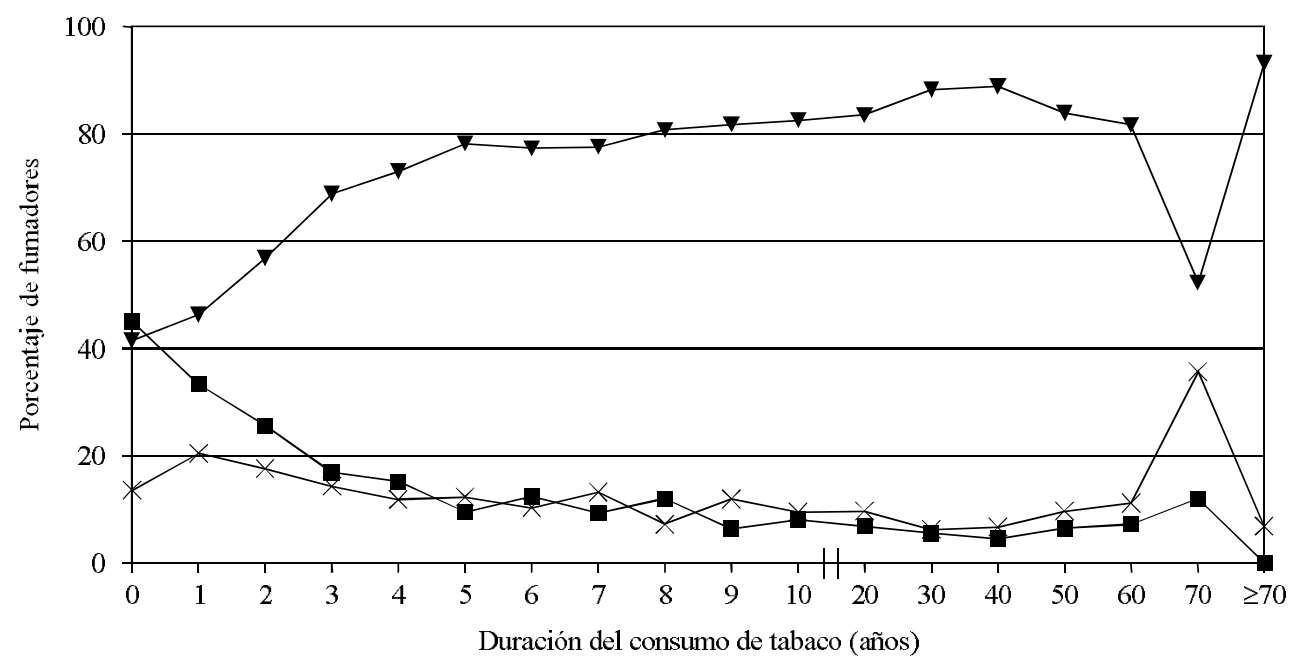

1b)

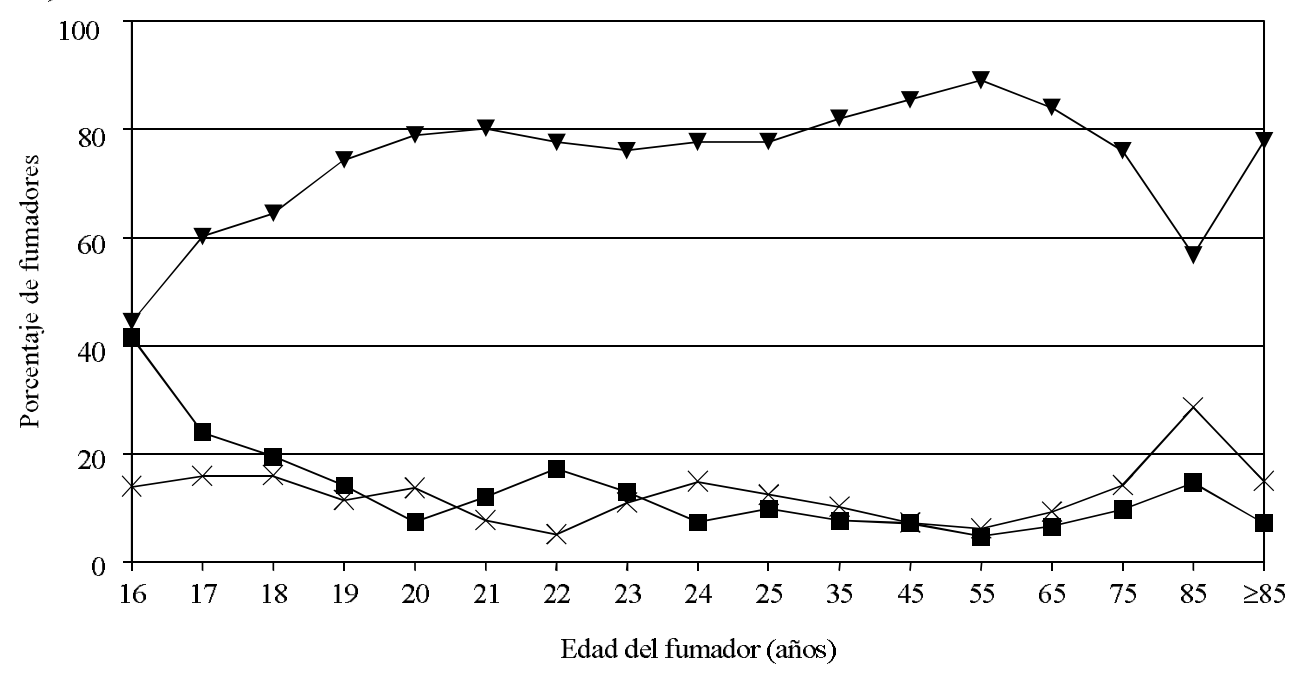

Fumadores ocasionales $\quad *$ Fumadores $\leq 5$ cigarrillos/día $\quad \rightarrow$ Fumadores $>5$ cigarrillos/día 
Estos resultados extienden a España los obtenidos en los fumadores ocasionales en otras poblaciones. En particular la menor duración e intensidad del consumo de tabaco de estos fumadores ${ }^{3}$, y la asociación a hábitos de vida más saludables que el consumo diario $^{31,32}$. Nuestro hallazgo de que los fumadores ocasionales son más frecuentes entre fumadores jóvenes y con menor duración de consumo de tabaco, y entre los más mayores y con duraciones de consumo más prolongadas ilustran también que el tabaquismo ocasional es un patrón relativamente típico de los que se inician ${ }^{31,33}$ y entre los que abandonan el hábito ${ }^{15}$. Además, ello no excluye que en cierta proporción de sujetos el consumo ocasional de tabaco sea durante largos periodos de tiempo (hábitos de consumo de 3 a 50 ó 60 años) una forma habitual o estable de consumo, ni que entre los fumadores ocasionales haya algunos sujetos que estén intentando dejar el tabaco o que acaben de recaer después de dejarlo ${ }^{4}$. Esto mismo podría ocurrir aunque con menos frecuencia en los fumadores más jóvenes y más ancianos ${ }^{31,34}$.

Entre las fortalezas de este estudio está el haberse realizado en una muestra de gran tamaño, representativa del conjunto de la población española. Entre sus limitaciones potenciales está el diseño transversal y que la información recogida es autoreportada. El diseño transversal impide establecer relaciones de causalidad ente las variables de estudio. Sin embargo, los objetivos del trabajo son fundamentalmente descriptivos, y sólo pretenden identificar características asociadas al patrón de consumo de tabaco que sean útiles para diseñar estrategias más específicas de control del tabaquismo y orienten sobre los grupos prioritarios de intervención. Por otro lado, hay evidencias de que es posible obtener por entrevista información válida sobre el patrón y la historia de consumo de tabaco ${ }^{35,36}$, el consumo de al$\mathrm{cohol}^{37,38}$, la actividad física ${ }^{36}$, o el nivel socioeconómico ${ }^{39}$.

Por último, nuestros resultados tienen implicaciones prácticas para el control del ta- baquismo, y sugieren que deben realizarse programas de investigación e intervención dirigidos de forma específica a los fumadores ocasionales. En primer lugar, ya que los fumadores ocasionales tienen mayor tasa de cesación tabáquica ${ }^{5,6}$, nuestros resultados muestran que en España hay una proporción sustancial de fumadores en los que las intervenciones son potencialmente más eficaces $\mathrm{y}$, por tanto, es prioritario intervenir. Este argumento se refuerza porque el consumo ocasional afecta sobre todo a mujeres que son las que en mayor proporción se incorporan al tabaquismo en España, y a jóvenes que pueden convertirse en fumadores diarios durante décadas. De hecho, la prevalencia de fumadores ocasionales es similar o superior a la magnitud del pequeño descenso de la prevalencia tabáquica ocurrido en España en los últimos años ${ }^{40}$. En segundo lugar, el mejor perfil de hábitos de vida relacionados con la salud de los fumadores ocasionales sugiere que la intervención sobre ellos podría tener beneficios adicionales a los de la cesación tabáquica, en concreto la reducción del desarrollo de hábitos más nocivos de salud como los que afectan a los fumadores diarios ${ }^{41}$. En tercer lugar, que el consumo ocasional de tabaco parezca ser un patrón estable de tabaquismo, muestra el interés de estudiar los determinantes concretos del mantenimiento del consumo de tabaco en fumadores de baja dependencia. Ello podrá orientar el consejo sanitario para dejar de fumar en estos sujetos.

\section{BIBLIOGRAFÍA}

1. U.S. Department of Health and Human Services. The Health Consequences of Smoking: Nicotine Addiction. A Report of the Surgeon General. Rockville, Maryland: U.S. Department of Health and Human Services, 1988. DHHS publication n. ${ }^{\circ}$ (CDC) 88-8406.

2. Shiffman S. Tobacco «chippers»- individual differences in tobacco dependence. Psychopharmacology 1989; 97: 539-47.

3. Gilpin E, Cavin SW, Pierce JP. Adult smokers who do not smoke daily. Addiction 1997; 92: 473-80. 
4. Evans NJ, Gilpin E, Pierce JP, Burns DM, Borland R, Johnson M, Bal D. Occasional smoking among adults: evidence from the California Tobacco Survey. Tob Control 1992; 1: 169-75.

5. Sargent JD, Mott LA, Stevens M. Predictors of smoking cessation in adolescents. Arch Pediatr Adolesc Med 1998; 152: 388-93.

6. Hennrikus DJ, Jeffery RW, Lando HA. The smoking cessation process: longitudinal observations in a working population. Prev Med 1995; 24: 235-44.

7. Luoto R, Uutela A, Puska P. Occasional smoking increases total and cardiovascular mortality among men. Nicotine and Tob Res 2000; 3: 133-9.

8. Doll R, Peto R. Cigarette smoking and bronchial carcinoma: dose and time relationships among regular and lifelong non-smokers. J Epidemiol Community Health 1978;32:303-13.

9. Goldstein J. The stigmatization of smokers: an empirical investigation. J Drug Educ 1991; 21: 167-82.

10. Hines D, Fretz AC, Nollen NL. Regular and occasional smoking by college students: personality attributions of smokers and nonsmokers. Psychol Rep 1998; 83: 1299-1306.

11. Del Río MC, Alvarez FJ. Patterns of smoking in Spain. Eur J Epidemiol 1994; 10: 595-8.

12. Ministerio de Sanidad y Consumo. Encuesta Nacional de Salud de España 1993. Madrid: Ministerio de Sanidad y Consumo; 1995.

13. Henríquez Sánchez $\mathrm{P}$, Alonso Bilbao JL, Beltrán Rodríguez R, Doreste Alonso J. Tabaquismo en Gran Canaria. Consumo y actitudes en adolescentes. Gac Sanit 2000; 14: 338-45.

14. Husten CG, McCarty MC, Giovino GA, Chrismon JH, Zhu BP. Intermittent smokers: a descriptive analysis of persons who have never smoked daily. Am J Public Health 1998; 88: 86-9.

15. Hennrikus DJ, Jeffery RW, Lando HA. Occasional smoking in a Minnesota working population. Am J Public Health 1996; 86: 1260-6.

16. Lindström M, Östergren PO. Intermittent and daily smokers: two different socioeconomic patterns, and diverging influence of social participation. Tob Control 2001; 10: 258-66.

17. Patterson RE, Haines PS, Popkin BM. Health lifestyle patterns of US Adults. Prev Med 1994; 23: 453-60.
18. Ma J, Betts NM, Hampl JS. Clustering of lifestyle behaviors: The relationship between cigarette smoking, alcohol consumption, and dietary intake. Am J Health Prom 2000; 15: 107-117.

19. Langlie JK. Interrelationships among preventive health behaviors: A test of competing hypotheses. Pub Health Rep 1979; 94: 216-25.

20. Johansson SE, Sundquist J. Change in lifestyle factors and their influence on health status and all-cause mortality. Int J Epidemiol 1999; 28: 1073-80.

21. Luoto R, Prättälä R, Uutela A, Puska P. Impact of unhealthy behaviors on cardiovascular mortality in Finland, 1978-1993. Prev Med 1998; 27: 93-100

22. Meng L, Maskarinec G, Lee J, Kolonel LN. Lifestyle factors and chronic diseases: application of a composite risk index. Prev Med 1999; 29. 296-304.

23. Bien TH, Burge R. Smoking and drinking: a review of literature. Int J Addict 1990; 25: 1429-54.

24. Shiffman S, Balabanis M. Associations between alcohol and tobacco. En: Fertig JB, Allen JP, editors. Alcohol and tobacco: from basic science to clinical practice. Bethesda, MD: National Institute of Health; 1995.p. 17-36.

25. Istvan J, Matarazo JD. Tobacco, alcohol, and caffeine use: A review of their interrelationships. Psychol Bull 1984; 95: 301-26.

26. Torabi MR, Bailey WJ, Majd-Jabbari M. Cigarette smoking as a predictor of alcohol and other drug use by children and adolescents: evidence of the «gateway drug effect». J Sch Health 1993; 63: 302-6.

27. Guallar Castillón P, Rodríguez Artalejo F, Díez Gañán L, Banegas Banegas JR, Lafuente Urdinguio P, Rey Calero J. El consumo de tabaco y la salud subjetiva en España. Med Clin (Barc) 2001; 116: $451-3$

28. Guallar-Castillón P, Rodríguez Artalejo F, Díez Gañán L, Banegas Banegas JR, Lafuente Urdinguio P, Herruzo Cabrera R. Consumption of alcoholic beverages and subjective health in Spain. J Epidemiol Community Health 2001; 55: 648-652.

29. Gutiérrez-Fisac JL, Guallar-Castillón, Díez Gañán L, López García E, Banegas Banegas JR, Rodríguez Artalejo F. Work-related physical activity is not associated with body mass index and obesity. Obes Res 2002; 10: 270-276.

Rev Esp Salud Pública 2002, Vol. 76, N. ${ }^{\circ} 4$ 
30. SAS/STAT Guide for personal computers, version 6.12. Cary (NC): SAS Institute; 1996.

31. Holmen TL, Barrett-Connor E, Holmen J, Bjermer L. Adolescent occasional smokers, a target group for smoking cessation? The Nord-Trondelag Health Study, Norway, 1995-1997. Prev Med 2000; 31: 682-90.

32. Resnicow K, Smith M, Harrison L, Drucker E. Correlates of occasional cigarette and marihuana use: are teens harm reducing? Addictive Behaviors 1999; 24: 251-66.

33. Patton GC, Carlin JB, Coffey C, Wolfe R, Hibbert M, Bowes G. The course of early smoking: a population-based cohort study over three years. Addiction 1998; 93: 1251-60.

34. Hines D, Nollen NL, Fretz AC. One-year follow-up of college students occasional smokers. Tob Control 1996; 5: 231-2.

35. Morabia A, Berstein MS, Curtin F, Berode M Validation of self-reported smoking status by simultaneous measurement of carbon monoxide and salivary thiocyanate. Prev Med 2001; 32: 82-8.
36. Bowling SJ, Morrill BD, Nafziger AN, Jenkins PL, Lewis C, Pearson TP. Validity of cardiovascular disease risk factors assessed by telephone survey: The Behavioral Risk Factor Survey. J Clin Epidemiol 1993; 46: 561-571.

37. Midanik L. The validity of self-reported alcohol consumption: a review of the literature. $\mathrm{Br} \mathrm{J}$ Addict 1982; 77: 357-382.

38. Gutiérrez-Fisac JL. Indicadores de consumo de alcohol en España. Med Clin (Barc) 1995; 104: 544-50.

39. Álvarez Dardet C, Alonso J, Domingo A, Regidor E. La medición de la clase social en ciencias de la salud. Informe de un grupo de trabajo de la Sociedad Española de Epidemiología. Barcelona: SG-Sociedad Española de Epidemiología; 1995.

40. Regidor E, Gutiérrez-Fisac JL. Indicadores de salud. Cuarta evaluación en España del programa regional europeo de Salud para Todos. Madrid: Ministerio de Sanidad y Consumo; 1999.

41. Laaksonen M, Luoto R, Helakorpi S, Uutela A. Associations between health-related behaviors: a 7 -year follow-up of adults. Prev Med 2002; 34 : 162-70. 